\title{
Matrix metalloproteinases (MMP-2,9) and their tissue inhibitors (TIMP-1,2) as novel markers of stress response and atherogenesis in children with chronic kidney disease (CKD) on conservative treatment
}

\author{
Kinga Musial · Danuta Zwolińska
}

Received: 14 April 2010 /Revised: 14 July 2010 /Accepted: 15 July 2010/Published online: 6 September 2010

(C) The Author(s) 2010. This article is published with open access at Springerlink.com

\begin{abstract}
The system of matrix metalloproteinases (MMPs) and their tissue inhibitors (TIMPs) may play a key role in atherogenesis of chronic kidney disease (CKD) patients by its impact on matrix accumulation. Connections with inflammation, stress, or endothelial dysfunction are also probable. However, the data on correlations between these parameters in CKD patients are scarce in adults and absent in children. The aim of our study was to evaluate serum concentrations of MMP-2, MMP-9, TIMP-1, and TIMP-2, as well as their correlations with markers of stress response (Hsp90- $\alpha$, anti-Hsp60), endothelial dysfunction (sE-selectin), and inflammation (high-sensitivity C-reactive protein) in CKD children treated conservatively. Thirtyseven patients were divided into two groups according to the CKD stage (gr.CKDI, 19 children with CKD stages 2-3; gr.CKDII, 18 subjects with CKD stages 4-5). Twentyfour age-matched healthy subjects served as controls. Serum concentrations of MMP-2, MMP-9, TIMP-1, TIMP-2, Hsp90- $\alpha$, anti-Hsp60, and sE-selectin were assessed by ELISA. Median values of MMP-2, MMP-9, TIMP-1, and TIMP-2 were significantly higher in all CKD children vs. controls and were increased in patients with CKD stages $4-5$ vs. CKD stages $2-3$. Hsp90- $\alpha$, antiHsp60, sE-selectin, and glomerular filtration rate predicted the values of MMPs and TIMPs. Chronic kidney disease in children is characterized by MMP/TIMP system dysfunction, aggravated by the progression of renal failure.
\end{abstract}

\footnotetext{
K. Musial • D. Zwolińska $(\square)$

Department of Pediatric Nephrology,

Wrocław Medical University,

M. Skłodowskiej-Curie 50/52,

50-369 Wrocław, Poland

e-mail: zwolin@nefped.am.wroc.pl
}

Correlations between examined parameters, heat shock proteins, and markers of endothelial damage suggest the possibility of MMP/TIMP application as indicators of stress response and atherogenesis in children with CKD on conservative treatment.

Keywords Autoimmunity · Heat shock proteins . Inflammation $\cdot$ Lipids $\cdot$ Matrix destruction

\section{Introduction}

Endothelial dysfunction, inflammation, dyslipidemia, and autoimmune reactions are key elements in the pathogenesis of atherosclerosis (Blasi 2008; Nilsson and Hansson 2008). Heat shock proteins (HSPs) and their antibodies also influence the process of atherosclerosis (Wick et al. 2004; Rigano et al. 2007). The best described example of such impact is that of Hsp60 and anti-Hsp60 working together. Anti-Hsp60 is generated in response to both bacterial and human Hsp60 and triggers autoimmune reactions against one's own HSPs (Pockley et al. 1999; Perschinka et al. 2003; Wu and Tanguay 2006). The impact of anti-Hsp60 on innate immunity is also projected by the activation of macrophages and by stimulation of nuclear factor (NF)- $\mathrm{kB}$, which is one of the regulators of matrix metalloproteinase (MMP) and tissue inhibitor of metalloproteinase (TIMP) secretion (Schett et al. 1995). Similar activity has been described very recently in the case of Hsp90- $\alpha$ (MadrigalMatute et al. 2010). Moreover, the role of HSPs in predicting risk of acute coronary syndrome and progression of atherosclerosis has also been confirmed (Dulin et al. 2010; Zhang et al. 2010). 
Accumulating data have pointed to the disturbed extracellular matrix metabolism in myocardial and vascular remodeling as being another new component of the atherosclerotic puzzle (Johnson et al. 2005, 2006; Kuzuya et al. 2006). Therefore, matrix metalloproteinases, endopeptidases with proteolytic activity, as well as their tissue inhibitors, have been proposed as a group of factors that add to the pathogenesis of atherosclerosis. Animal models and in vitro investigations have shown their multifaceted actions, varying from protective and antiatherogenic in the case of TIMP-2, through neutral of TIMP-1 or ambiguous of MMP-9, to proatherogenic of MMP-2 (Luttun et al. 2004; de Nooijer et al. 2006; Johnson et al. 2005, 2006; Kuzuya et al. 2006).

Moreover, gelatinases A and B (MMP-2 and MMP-9) occupy an established position among risk factors for myocardial infarction (Jefferis et al. 2010) and as predictors of mortality due to acute coronary syndrome (Dhillon et al. 2010), whereas their tissue inhibitors TIMPs have an impact on postmyocardial infarction remodeling (Kandalam et al. 2010) and correlate positively with left ventricular mass and wall thickness (Hansson et al. 2009). The role of MMPs in kidney disease has been studied extensively (Catania et al. 2007), and special attention has been paid to the impact on ischemic acute renal injury and scarring in the course of glomerulopathies (Caron et al. 2005; Cheng et al. 2006; Johnson et al. 2002). However, the data on their role in chronic kidney disease, characterized by accelerated progression of atherosclerosis, are scarce and come mainly from adult patients on hemodialysis (Preston et al. 2002; Pawlak et al. 2007).

There are no data so far on MMPs and TIMPs in pediatric patients with chronic kidney disease on conservative treatment. Therefore, the first aim of our study was to evaluate the levels of MMP-2, MMP-9, TIMP-1, and TIMP-2 in serum samples from children in different stages of chronic kidney disease treated conservatively. The second goal was to analyze whether there is any relationship between those parameters and other factors predisposing to atherosclerosis, such as disturbed stress response (Hsp90- $\alpha$, anti-Hsp60), endothelial activation (sE-selectin), inflammation (high-sensitivity C-reactive protein (hsCRP)), or dyslipidemia.

\section{Subjects and methods}

Sixty-one patients enrolled in the study were divided into three groups.

The first group (chronic kidney disease (CKD) I) consisted of 19 patients (10 girls, nine boys, median age of 8.5 years, interquartile range of $4.5-15$ years) with CKD stages 2-3 treated conservatively (median glomerular filtration rate (GFR) calculated according to the Schwartz formula $51 \mathrm{ml} / \mathrm{min}$ per $1.73 \mathrm{~m}^{2}$ ). The factors causing CKD were: reflux nephropathy (seven cases), chronic glomerulonephritis (five), chronic pyelonephritis (one), polycystic kidney disease (four), hemolytic uremic syndrome (one), and cystinosis (one).

The second group (CKD II) contained 18 patients (10 girls, eight boys; median age of 11 years, interquartile range of 5-17.5 years) with CKD stages $4-5$ on conservative treatment (median GFR $23 \mathrm{ml} / \mathrm{min}$ per $1.73 \mathrm{~m}^{2}$ ). Primary diseases causing CKD were reflux nephropathy (nine cases), chronic glomerulonephritis (six), lupus nephropathy (one), neurogenic bladder (one), hemolytic uremic syndrome (one). In all patients, phosphate binders and vitamin D metabolites were supplemented.

Twenty-four children (13 girls, 11 boys, median age of 10.5 years, range of $5-16.5$ years) with primary nocturnal enuresis, with normal kidney function, served as controls.

None of the patients showed clinical evidence of infection, malignancy, or vasculitis, suffered from diabetes, smoked, and took antibiotics, corticosteroids, or immunosuppressive therapy. All the CKD children had blood pressure values below the 90th percentile for smaller children and below 120/80 mmHg for adolescents, according to the criteria of the fourth report on high blood pressure in children and adolescents (National 2004). CKD stage 2-3 children, except for three subjects receiving angiotensin-converting enzyme (ACE) inhibitors in nephroprotective doses, did not require antihypertensive drugs. In the CKD stage 4-5 group blood pressure was well controlled either without medication (13 children) or with the use of ACE inhibitors (three patients) or ARB (one child).

Informed consent was obtained from the subjects and their parents, if necessary. The research project was approved by the University ethics committee, in accordance with the Helsinki declaration.

Blood samples were drawn from peripheral veins after an overnight fast. Samples were clotted for $30 \mathrm{~min}$ and centrifuged at $4^{\circ} \mathrm{C}$ for $10 \mathrm{~min}$, and then serum was stored at $-20^{\circ} \mathrm{C}$ until assayed. Serum concentrations of MMP-2 (gelatinase A), MMP-9 (gelatinase B), TIMP-1, TIMP-2, sE-selectin, Hsp90- $\alpha$, and anti-Hsp60 were evaluated by commercially available ELISA kits (Stressgen, R\&D Systems, UK). In the case of MMP/TIMP, standards and serum samples were transferred to 96-well microplates precoated with recombinant antibodies to human MMP-2, MMP-9, TIMP-1, and TIMP-2. Each sample was tested in duplicate, and the arithmetical mean was considered a final result. Measurements were performed according to the manufacturer's instructions; results were calculated by reference to standard curves. 
The methods of evaluation of Hsp90- $\alpha$, anti-Hsp60, and sE-selectin were described in our previous publication (Musiał et al. 2010).

In all patients, the kidney function was assessed, calculated by the Schwartz formula. The lipid profile (total cholesterol, high-density lipoprotein (HDL) cholesterol, low-density lipoprotein (LDL) cholesterol, triglycerides by BioSystems, Barcelona, Spain) and hsCRP as a marker of inflammation (nephelometry by Dade Behring, Marburg, Germany) were also evaluated.

\section{Statistical analysis}

Results are expressed as median values and interquartile ranges. Differences between all groups were evaluated using nonparametric tests (Kruskal-Wallis, Mann-Whitney $U$ ). The relations between parameters were assessed by linear regression analysis. The linear regression equations were calculated as $y=\beta \times+a$ (y, dependent variable; $\beta$, regression coefficient; $x$, independent variable; $a$, constant term). We presented only those equations where both regression coefficient and constant term were statistically significant. Statistical analysis was performed using the package Statistica ver. 8.0. A $p$ value of $<0.05$ was considered significant.

\section{Results}

MMP-2 and MMP-9

MMP-2 and MMP-9 concentrations in all CKD children were significantly higher vs. controls $(p<0.000001)$, and the levels in the CKD stage 4-5 subgroup were increased when compared to CKD stages 2-3 (Fig. 1a, b).

\section{TIMP-1 and TIMP-2}

In the case of TIMP-1, the concentrations in the CKD group were also elevated vs. controls $(p<0.001)$. However, the median TIMP-1 values in the CKD stages 2-3 were comparable, whereas in the CKD stages 4-5 they were higher than in the control group (Fig. 1c). TIMP-2 levels were increased in CKD children vs. controls $(p<0.000001)$ and rose with the progression of renal failure (Fig. 1d).

\section{MMP/TIMP ratios}

When MMP-9/TIMP-1 and MMP-2/TIMP-2 ratios were assessed, the first was significantly higher $(p<0.000001)$ and the second was significantly lower $(p<0.000001)$ in all CKD children than in the control group. However, none of them could differentiate between the CKD stage 2-3 and the CKD stage 4-5 groups (Fig. 1e, f).
Hsp90- $\alpha$, anti-Hsp60, sE-selectin

Hsp90- $\alpha$, anti-Hsp60, and sE-selectin serum concentrations in the CKD population were significantly higher than in the control group (Table 1).

hsCRP, lipid profile

High-sensitivity CRP and total cholesterol levels did not show significant differences between CKD children and controls (Table 1). HDL concentrations were increased, whereas those of LDL and triglycerides were decreased in CKD patients versus controls (Table 1).

\section{Linear regression analysis}

In all CKD children, MMPs and TIMPs correlated with each other. We have also found correlations between MMPs, TIMPs, and other examined parameters. In detail, Hsp90- $\alpha$, anti-Hsp60, sE-selectin, and GFR predicted the values of MMP-2, MMP-9, and TIMP-1 (Table 2). In the case of TIMP-1, such prediction concerned sE-selectin and GFR (Table 2).

No associations between MMP/TIMP, the lipid profile, and hsCRP were observed.

\section{Discussion}

In our study, we have shown for the first time the dysfunctional MMP/TIMP system and its correlation to markers of stress response in children with chronic kidney disease treated conservatively.

Serum MMP-2 concentrations in the examined population were elevated, when compared to controls, similar to the results obtained in adults by others (Peiskerova et al. 2009; Chang et al. 2006). The novelty of our observation was the fact that MMP-2 levels increased gradually with the progression of CKD and correlated with GFR. Although Chang et al. (2006) have previously reported the correlation between gelatinases and serum creatinine, the interpretation of these results should be viewed with caution due to methodological inconsistencies (e.g., plasma collection in ethylenediaminetetraacetic acid (EDTA)-containing tubes, although the use of EDTA is not recommended by the manufacturer due to its chelating properties). We have also revealed the correlations between MMP-2, stress response, and endothelial activation in the pediatric CKD population. These findings add new data to the knowledge of cardiovascular complication pathogenesis in CKD children (Mitsnefes 2005). Since MMP-2 has recently been defined as a predictor of mortality in acute coronary syndrome (Dhillon et al. 2010) and its correlation with arterial 
a

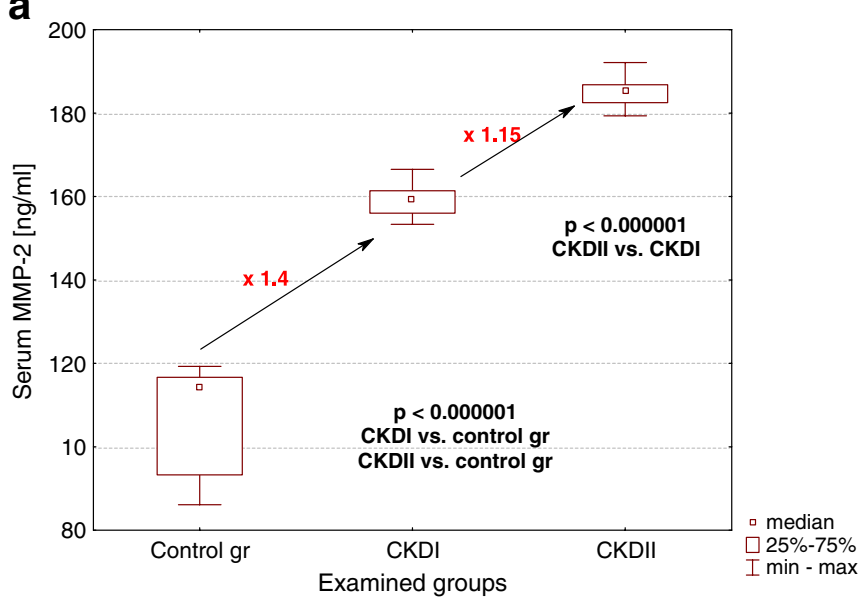

C

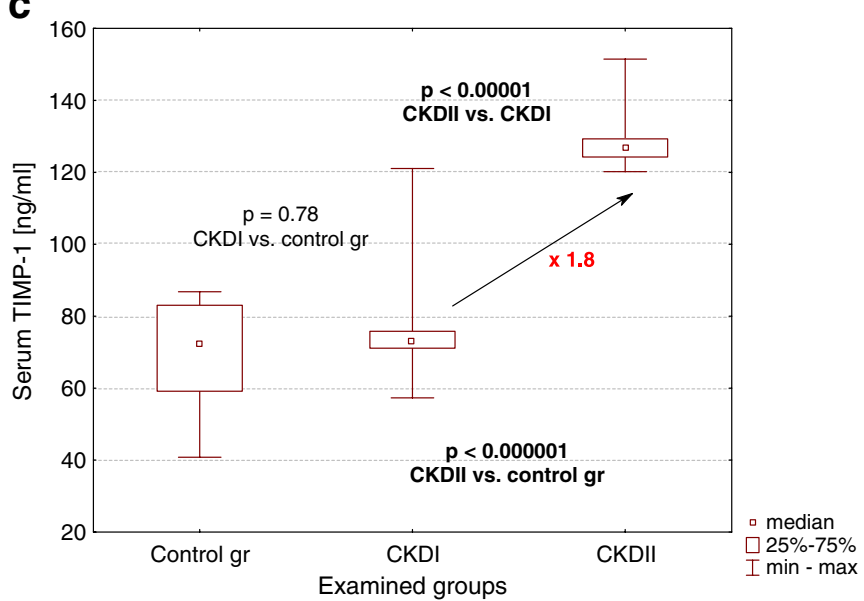

e

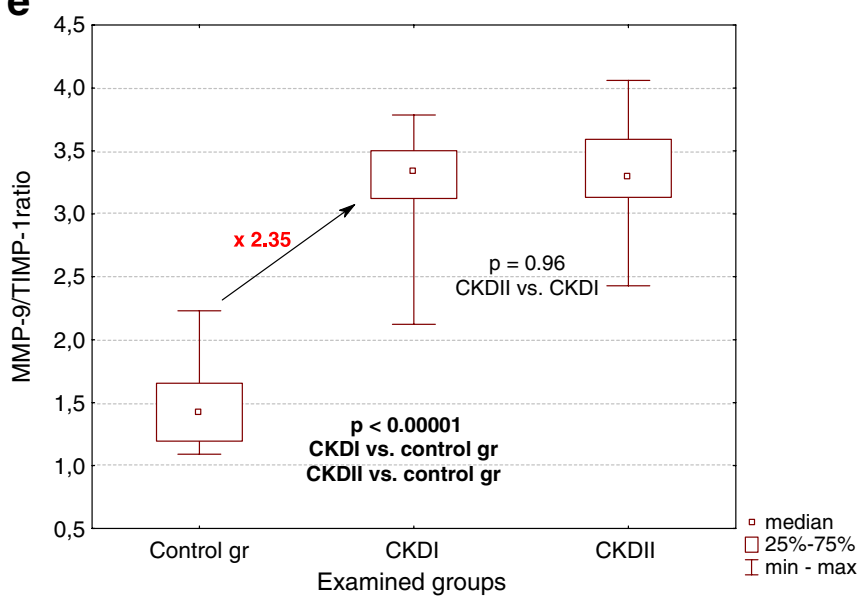

b

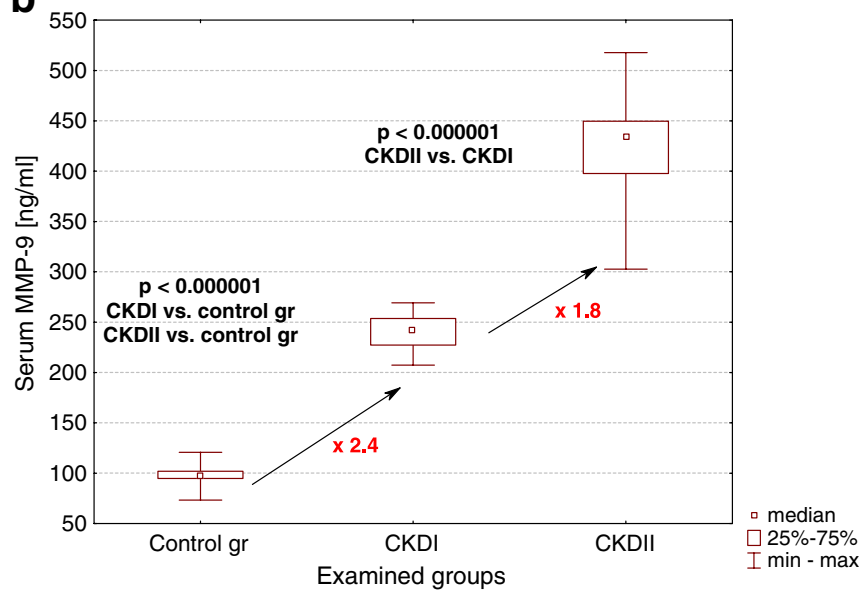

d

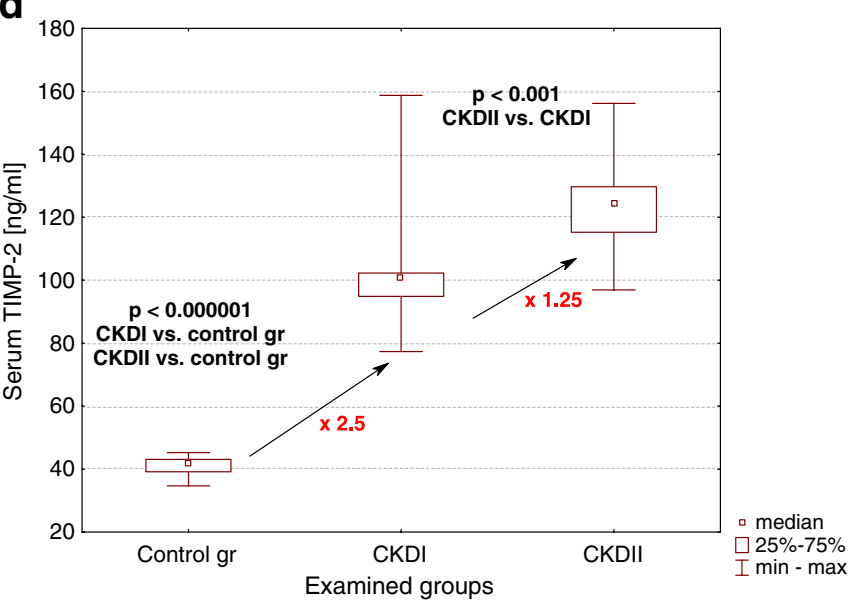

f

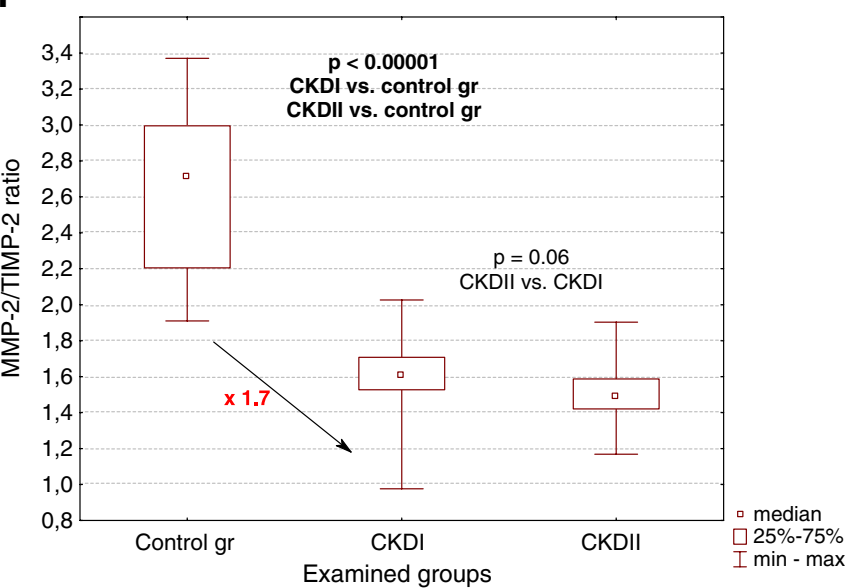

Fig. 1 Serum concentrations of examined parameters (a MMP-2; b MMP-9; $\mathbf{c}$ TIMP-1; d TIMP-2; e MMP-9/TIMP-1; f MMP-2/TIMP-2) in the CKD children and in the control group

stiffness in CKD patients has been revealed (Chung et al. 2009), the usefulness of gelatinase $A$ as one of the potential markers of atherogenesis in children with CKD seems justified.

The data on MMP-9 concentrations in CKD patients are scarce and contradictory and concern only adults. Chang et al. (2006) found decreased levels of gelatinase B, whereas Peiskerova et al. (2009) noticed no difference between controls and CKD patients. In our group, a gradual increase was observed, together with an inverse correlation to GFR values. Such discrepancies may result from methodology or differences in the profile of examined 
Table 1 The median values and interquartile ranges of examined parameters in the patients with $\mathrm{CKD}$ and in the control group

$N S$ nonsignificant

${ }^{*} p<0.01 ; * * p<0.001 ; * * * p<$ $0.0001 ; * * * * p<0.00001 \mathrm{CKD}$ vs. controls

\begin{tabular}{lll}
\hline Parameter & \multicolumn{2}{l}{ Median values (lower-upper quartile) of analyzed parameters } \\
\cline { 2 - 3 } & $\begin{array}{l}\text { Control group } \\
n=24\end{array}$ & $\begin{array}{l}\text { CKD patients } \\
n=37\end{array}$ \\
\hline Hsp90- $\alpha(\mathrm{ng} / \mathrm{ml})$ & $5.0(4.5-5.5)$ & $20.0(15.0-25.0)^{* * * *}$ \\
Anti-Hsp60 $(\mathrm{ng} / \mathrm{ml})$ & $11.3(8.2-26.7)$ & $46.0(14.9-81.7)^{*}$ \\
sE-selectin $(\mathrm{ng} / \mathrm{ml})$ & $26.1(22.2-28.0)$ & $68.0(54.0-80.0)^{* * * *}$ \\
hsCRP $(\mathrm{mg} / \mathrm{l})$ & $0.33(0.21-0.63)$ & $0.28(0.16-0.81) \mathrm{NS}$ \\
CHOL $(\mathrm{mg} / \mathrm{dl})$ & $188.0(181.0-196.0)$ & $193.0(167.8-235.3) \mathrm{NS}$ \\
HDL $(\mathrm{mg} / \mathrm{dl})$ & $62.0(59.0-67.0)$ & $52.0(49.5-61.0)^{* *}$ \\
LDL $(\mathrm{mg} / \mathrm{dl})$ & $99.5(91.0-109.0)$ & $100.0(95.0-147.0)^{* *}$ \\
TGL $(\mathrm{mg} / \mathrm{dl})$ & $86.0(66.5-100.0)$ & $115.0(100.0-159.0)^{* * *}$ \\
\hline
\end{tabular}

groups. In detail, the CKD population examined by Chang et al. (2006) contained adults with mean creatinine clearance $(\mathrm{CCr})$ of $11 \mathrm{ml} / \mathrm{min}$ and was divided into subgroups with $\mathrm{CCr}$ less than $10 \mathrm{ml} / \mathrm{min}$, between 10 and $15 \mathrm{ml} / \mathrm{min}$, and over $15 \mathrm{ml} / \mathrm{min}$. Our patients had relatively higher GFR values (CKD 2-3 $51 \mathrm{ml} / \mathrm{min}$; CKD 4-5 $23 \mathrm{ml} /$ $\mathrm{min}$ ), and we had no subjects with $\mathrm{CCr}$ less than $15 \mathrm{ml} / \mathrm{min}$. Another reason for difficulties in the interpretation of MMP-9 elevation may be the fact of its ambiguous activity in in vitro investigations. Gelatinase B was described as protecting plaque stability in its early stage and increasing its vulnerability in the case of advanced lesion (Luttun et al. 2004; de Nooijer et al. 2006).

Moreover, matrix metalloproteinases are subject to different factors modifying their activity. The levels of MMPs depend on the presence of diabetic nephropathy (Rysz et al. 2007), hypercholesterolemia (El Messal et al. 2006), inflammation (Addabbo et al. 2007), increased oxidative stress (Valentin et al. 2005; Castro et al. 2009),
ACE inhibitors (Lods et al. 2003), and hypertension (Castro et al. 2008). Although we did not observe the correlation of examined enzymes with classical indicators of atherosclerotic changes, such as lipids or hsCRP, we have added sEselectin, a marker of endothelial dysfunction, and heat shock proteins, to that list. Moreover, serum Hsp90- $\alpha$, antiHsp60, sE-selectin, and GFR predicted the concentrations of MMPs and TIMPs in all CKD children. Therefore, it is probable that in the pediatric CKD population metalloproteinases share common features with stress markers rather than with those typical for atherosclerosis. The enzyme levels, increasing progressively with aggravation of kidney failure, stress conditions, and toxemia, would seem to confirm such a theory. However, correlations of MMPs and TIMPS with Hsp90- $\alpha$ and anti-Hsp60 in CKD patients are new findings, not confirmed by any earlier study. The possible explanation for such links might lie in the common points of signaling pathway regulation. Both Hsp90- $\alpha$ and Hsp60 increase secretion of cytokines (e.g.,

Table 2 The statistically significant correlations between the examined parameters assessed by linear regression analysis in the CKD patients

\begin{tabular}{|c|c|c|c|c|c|}
\hline Dependent variable & Independent variable & Regression coefficient $\beta$ & Constant term & $95 \%$ confidence interval $(\mathrm{CI})$ & $p$ \\
\hline \multicolumn{6}{|l|}{ CKD patients } \\
\hline MMP-2 (ng/ml) & Hsp90- $\alpha(\mathrm{ng} / \mathrm{ml})$ & -1.18 & 196.28 & $169.09-176.22$ & 0.00 \\
\hline MMP-2 (ng/ml) & Anti-Hsp60 (ng/ml) & -0.13 & 179.31 & $168.96-177.96$ & 0.02 \\
\hline MMP-2 (ng/ml) & sE-selectin (pg/ml) & 0.70 & 123.93 & $168.60-174.59$ & 0.00 \\
\hline MMP-2 (ng/ml) & GFR (ml/min) & -0.49 & 186.93 & $166.64-177.58$ & 0.00 \\
\hline MMP-9 (ng/ml) & Hsp90- $\alpha$ (ng/ml) & -8.82 & 513.79 & $311.73-362.88$ & 0.00 \\
\hline MMP-9 (ng/ml) & Anti-Hsp60 (ng/ml) & -0.85 & 381.53 & $308.97-375.92$ & 0.03 \\
\hline MMP-9 (ng/ml) & sE-selectin (pg/ml) & 4.60 & 17.42 & $305.03-355.08$ & 0.00 \\
\hline MMP-9 (ng/ml) & GFR (ml/min) & -3.99 & 460.64 & $301.59-379.89$ & 0.00 \\
\hline TIMP-1 (ng/ml) & Hsp90- $\alpha[\mathrm{ng} / \mathrm{ml}]$ & -2.20 & 147.27 & $95.09-111.35$ & 0.00 \\
\hline TIMP-1 (ng/ml) & Anti-Hsp60 (ng/ml) & -0.29 & 118.59 & $95.79-114.40$ & 0.01 \\
\hline TIMP-1 (ng/ml) & sE-selectin (pg/ml) & 1.53 & -2.89 & 95.09-106.95 & 0.00 \\
\hline TIMP-1 (ng/ml) & GFR (ml/min) & -1.17 & 137.92 & $91.98-113.91$ & 0.00 \\
\hline TIMP-2 (ng/ml) & sE-selectin $[\mathrm{pg} / \mathrm{ml}]$ & 0.54 & 75.71 & $106.22-118.67$ & 0.01 \\
\hline TIMP-2 (ng/ml) & GFR (ml/min) & -0.58 & 128.80 & $91.98-113.91$ & 0.03 \\
\hline
\end{tabular}


TNF- $\alpha$ ) and adhesion molecules (e.g., sE-selectin) and trigger NF- $\mathrm{KB}$ activity, which then upregulate the synthesis of MMPs and TIMPs (Zhang et al. 1998; Kol et al. 1999; Businaro et al. 2009; Madrigal-Matute et al. 2010). Therefore, there is a functional chain suggesting the reason for the observed correlations. Such connection has not been proven in the case of TIMP-2, thus explaining the lack of relation between Hsp90- $\alpha$ and anti-Hsp60 and TIMP-2 in our population. The question whether any direct regulation between HSP and MMP/TIMP system takes place, although intriguing, requires further detailed investigation.

We have also revealed that all examined MMPs and TIMPs correlate with each other, thus aggravating the complexity of interrelations between them and forming a kind of functional network including processes engaged in reactions to stress conditions.

TIMP-1 concentrations remained unchanged in children with CKD stages $2-3$ and increased with disease progression, being higher in CKD stages 4-5 than in controls and in CKD stages 2-3. As far as we are concerned, there are no available data on other studies evaluating TIMP-1 in various CKD stages. One of the possible explanations is that TIMP-1 elevation, seen barely in advanced renal failure, is a compensatory response to MMP-9 increase in early CKD stages. Secondly, according to the hypothesis of Newby (2008), the prevalence of MMP over TIMP activity might result from the fact that in the early stages of atherosclerosis, when monocytes migrate across the endothelial monolayer, mainly MMPs are needed to facilitate the penetration of cells. Subsequently, the TIMP overactivity would be more evident when the in situ differentiation of monocytes into macrophages takes place. The TIMP-2 high concentrations, seen already in CKD stages 2-3, would suggest the early response to the increase of MMP-2, known for its proatherogenic activity. Thus, the progressive elevation in CKD stages 4-5 would be a consequence of further MMP-2 elevation.

However, the best way to evaluate the net effect of balance between MMPs and their inhibitors was to assess the values of MMP/TIMP ratios. Both MMP-9/TIMP-1 and MMP-2/TIMP-2 ratio values in CKD patients differed significantly from those in controls. In the case of the MMP-9/TIMP-1 ratio, MMP-9 increased 2.4-fold in CKD stage 2-3 patients vs. controls, whereas TIMP-1 did not, causing the net 2.4-fold rise of the ratio value. When children in CKD stages 2-3 and CKD stages 4-5 were compared, both MMP-9 and TIMP-1 increased 1.8-fold, so the ratio did not change. Taken together, the MMP-9 response dominated over that of TIMP-1 only in the early $\mathrm{CKD}$, and there was no difference between stages 2-3 and 4-5.

When the MMP-2/TIMP-2 ratio was taken into account, both MMP-2 and TIMP-2 concentrations were higher in CKD stages 2-3 vs. controls. The elevation of protective
TIMP-2 was more pronounced (2.5-fold) than that of proatherogenic MMP-2 (1.4-fold), thus causing the net decrease of a ratio value. In CKD stages 4-5, when compared to stages $2-3$, the rise of both MMP-2 and TIMP-2 was proportional and the ratio value, as in the case of MMP-9/TIMP-1, did not change. Therefore, it seems that disturbances in MMP/TIMP balance are already noticeable in early $\mathrm{CKD}$, whereas their correction and stabilization take place in later stages of renal failure.

\section{Conclusions}

Our study describes for the first time the network of MMP/ TIMP systems in children with chronic kidney disease. Relationships between these parameters, heat shock proteins, and markers of endothelial dysfunction suggest the possible role of MMPs and TIMPs in the stress response and atherogenesis of CKD children. The progressive changes in concentrations of MMPs and their inhibitors may illustrate the adaptive response to unfavorable conditions resulting from chronic dysfunction of the kidney. However, the precise role of MMP/TIMP balance and its relation to the stress response in the CKD pediatric population need further investigation.

Open Access This article is distributed under the terms of the Creative Commons Attribution Noncommercial License which permits any noncommercial use, distribution, and reproduction in any medium, provided the original author(s) and source are credited.

\section{References}

Addabbo F, Mallamaci F, Leonardis D et al (2007) Searching for biomarker patterns characterizing carotid atherosclerotic burden in patients with reduced renal function. Nephrol Dial Transplant 22:3521-3526

Blasi C (2008) The autoimmune origin of atherosclerosis. Atherosclerosis 201:17-32

Businaro R, Profumo E, Tagliani A et al (2009) Heat-shock protein 90: a novel autoantigen in human carotid atherosclerosis. Atherosclerosis 207:74-83

Caron A, Desrosiers RR, Beliveau R (2005) Ischemia injury alters endothelial cell properties of kidney cortex: stimulation of MMP9. Exp Cell Res 310:105-116

Castro MM, Rizzi E, Figueiredo-Lopes L et al (2008) Metalloproteinase inhibition ameliorates hypertension and prevents vascular dysfunction and remodeling in renovascular hypertensive rats. Atherosclerosis 198:320-331

Castro MM, Rizzi E, Rodrigues GJ et al (2009) Antioxidant treatment reduces matrix metalloproteinase-2-induced vascular changes in renovascular hypertension. Free Rad Biol Med 46:1298-1307

Catania JM, Chen G, Parrish AR (2007) Role of matrix metalloproteinases in renal pathophysiologies. Am J Physiol Ren Physiol 292:F905-F911 
Chang HR, Yang SF, Li ML, Lin ChCh, Hsieh YS, Lian JD (2006) Relationships between circulating matrix metalloproteinase-2 and -9 and renal function in patients with chronic kidney disease. Clin Chim Acta 366:243-248

Cheng S, Pollock AS, Mahimkar R, Olson JL, Lovett DH (2006) Matrix metalloproteinase 2 and basement membrane integrity: a unifying mechanism for progressive renal injury. FASEB J 20: E1248-E1256

Chung AW, Yang HH, Kim JM et al (2009) Upregulation of matrix metalloproteinase-2 in the arterial vasculature contributes to stiffening and vasomotor dysfunction in patients with chronic kidney disease. Circulation 120:792-801

de Nooijer R, Verkleij CJN, von der Thusen HJ et al (2006) Lesional overexpression of matrix metalloproteinase- 9 promotes intraplaque hemorrhage in advanced lesions but not earlier stages of atherogenesis. Arterioscler Thromb Vasc Biol 26:340-346

Dhillon OS, Khan SQ, Narayan HK et al (2010) Matrix metalloproteinase-2 predicts mortality in patients with acute coronary syndrome. Clin Sci 118:249-257

Dulin E, Garcia-Barreno P, Guisasola MC (2010) Extracellular heat shock protein 70 (HSPA1A) and classical vascular risk factors in a general population. Cell Stress Chaperones. doi:10.1007/ s12192-010-0201-2

El Messal M, Beaudeux JL, Drissi A et al (2006) Elevated serum levels of proinflammatory cytokines and biomarkers of matrix remodeling in never-treated patients with familial hypercholesterolemia. Clin Chim Acta 366:185-189

Hansson J, Lind L, Hulthe J, Sundstrom J (2009) Relations of serum MMP-9 and TIMP-1 levels to left ventricular measures and cardiovascular risk factors: a population-based study. Eur J Cardiovasc Prev Rehab 16:297-303

Jefferis BJ, Whincup P, Welsh P et al (2010) Prospective study of matrix metalloproteinase- 9 and risk of myocardial infarction and stroke in older men and women. Atherosclerosis 208:557-563

Johnson TS, Haylor JL, Thomas GL et al (2002) Matrix metalloproteinases and their inhibitors in experimental renal scarring. Exp Nephrol 10:182-195

Johnson JL, George SJ, Newby AC et al (2005) Divergent effects of matrix metalloproteinases $3,7,9$ and 12 on atherosclerotic plaque stability in mouse brachiocephalic arteries. PNAS 102:15575-15580

Johnson JL, Baker AH, Oka K et al (2006) Suppression of atherosclerotic plaque progression and instability by tissue inhibitor of metalloproteinase-2. Involvement of macrophage migration and apoptosis. Circulation 113:2435-2444

Kandalam V, Basu R, Abraham T et al. (2010) TIMP-2 deficiency accelerates adverse post-myocardial infarction remodeling because of enhanced MT1-MMP activity despite lack of MMP2 activation. Circ Res (in press)

Kol A, Bourcier T, Lichtman AH, Libby P (1999) Chlamydial and human heat shock protein 60 s activate human vascular endothelium, smooth muscle cells, and macrophages. J Clin Invest 103:571-577

Kuzuya M, Nakamura K, Sasaki T et al (2006) Effect of MMP-2 deficiency on atherosclerotic lesion formation in apoE-deficient mice. Arterioscler Thromb Vasc Biol 26:1120-1125

Lods N, Ferrari P, Frey FJ et al (2003) Angiotensin-converting enzyme inhibition but not angiotensin II receptor blockade regulates matrix metalloproteinase activity in patients with glomerulonephritis. J Am Soc Nephrol 14:2861-2872

Luttun A, Lutgens E, Manderveld A et al (2004) Loss of matrix metalloproteinase- 9 or matrix metalloproteinase- 12 protects apolipoprotein E-deficient mice against atherosclerotic media destruction but differentially affects plaque growth. Circulation 109:1408-1414
Madrigal-Matute J, Lopez-Franco O, Blanco-Colio LM et al (2010) Heat shock protein 90 inhibitors attenuate inflammatory responses in atherosclerosis. Cardiovasc Res. doi:10.1093/cvr/crq046

Mitsnefes MM (2005) Cardiovascular disease in children with chronic kidney disease. Adv Chron Kidney Dis 12:397-405

Musiał K, Szprynger K, Szczepańska M, Zwolińska D (2010) The heat shock protein profile in children with chronic kidney disease. Perit Dial Int 30:227-232

National High Blood Pressure Education Program Working Group on High Blood Pressure in Children and Adolescents (2004) The fourth report on the diagnosis, evaluation, and treatment of high blood pressure in children and adolescents. Pediatrics 114:555576

Newby AC (2008) Metalloproteinase expression in monocytes and macrophages and its relationship to atherosclerotic plaque stability. Arterioscler Thromb Vasc Biol 28:2108-2114

Nilsson J, Hansson GK (2008) Autoimmunity in atherosclerosis: a protective response losing control? J Int Med 263:464-478

Pawlak K, Pawlak D, Myśliwiec M (2007) Serum matrix metalloproteinase-2 and increased oxidative stress are associated with carotid atherosclerosis in hemodialyzed patients. Atherosclerosis 190:199-204

Peiskerova M, Kalousova M, Kratochvilova M et al (2009) Fibroblast growth factor 23 and matrix-metalloproteinases in patients with chronic kidney disease: are they associated with cardiovascular disease? Kidney Blood Press Res 32:276-283

Perschinka H, Mayr M, Millonig G, Mayerl Ch, Van der Zee R, Morrison SG, Morrison RP, Xu Q, Wick G (2003) Crossreactive $\mathrm{B}$-cell epitopes of microbial and human heat shock protein $60 / 65$ in atherosclerosis. Arterioscler Thromb Vasc Biol 23:1060-1065

Pockley AG, Bulmer J, Hanks BM, Wright BH (1999) Identification of human heat shock protein 60 (Hsp60) and anti-Hsp60 antibodies in the peripheral circulation of normal individuals. Cell Stress Chaperones 4:29-35

Preston GA, Barrett CV, Alcorta DA et al (2002) Serum matrix metalloproteinases MMP-2 and MMP-3 levels in dialysis patients vary independently of CRP and IL-6 levels. Nephron 92:817823

Rigano R, Profumo E, Buttari B et al (2007) Heat shock proteins and autoimmunity in patients with carotid atherosclerosis. Ann NY Acad Sci 1107:1-10

Rysz J, Banach M, Stolarek RA et al (2007) Serum matrix metalloproteinases MMP-2 and MMP-9 and metalloproteinase tissue inhibitors TIMP-1 and TIMP-2 in diabetic nephropathy. J Nephrol 20:444-452

Schett G, Xu Q, Amberger A, Van der Zee R, Recheis H, Willeit J, Wick G (1995) Autoantibodies against heat shock protein 60 mediate endothelial cytotoxicity. J Clin Invest 96:2569-2577

Valentin F, Bueb JL, Kieffer P et al (2005) Oxidative stress activates MMP-2 in cultured human coronary smooth muscle cells. Fundam Clin Pharmacol 19:661-667

Wick G, Knoflach M, Xu Q (2004) Autoimmune and inflammatory mechanisms in atherosclerosis. Annu Rev Immunol 22:361-403

Wu T, Tanguay RM (2006) Antibodies against heat shock proteins in environmental stresses and diseases: friend or foe? Cell Stress Chaperones 11:1-12

Zhang Y, McCluskey K, Fujii K, Wahl LM (1998) Differential regulation of monocyte matrix metalloproteinase and TIMP-1 production by TNF- $\alpha$, granulocyte-macrophage CSF, and IL-1 $\beta$ through prostaglandin-dependent and -independent mechanisms. J Immunol 161:3071-3076

Zhang X, Xu Z, Zhou L et al (2010) Plasma levels of Hsp70 and antiHsp70 antibody predict risk of acute coronary syndrome. Cell Stress Chaperones. doi:10.1007/s12192-010-0180-3 